Conclusion. Engagement with the commissioned training was encouraging. Respondents were, on average, relatively confident in conducting capacity assessment, but considerable variation in confidence, and a lower confidence in completing medical reports. This might suggest that some may require further training. A poor response rate among non-psychiatrists indicates potential respondent bias in favour of those already more cognisant of capacity in routine practice. A correlation between more practiced assessors and anticipated impact on service provision could suggest that some clinicians may be underestimating the potential impact of DoLS; the same groups should therefore be resurveyed after DoLS implementation.

\section{A framework for nurturing doctors: systematic review of wellbeing interventions in medical students}

Rhian Bradley ${ }^{1 \star}$, Tahmina Yousofi ${ }^{1}$, Rafey Faruqui ${ }^{1}$ and Kate Hamilton-West ${ }^{2}$

${ }^{1}$ Kent and Medway NHS and Social Care Partnership Trust and

${ }^{2}$ University of Kent

${ }^{*}$ Corresponding author.

doi: 10.1192/bjo.2021.641

Aims. UK medical students report high levels of stress, in particular within the coronavirus pandemic: $46 \%$ have a probable psychiatric disorder; almost $15 \%$ consider suicide; $80 \%$ describe support as poor or moderately adequate. Our aim was to propose a novel conceptual framework for the implementation of effective interventions to reduce their stress and support wellbeing.

Method. A systematic review of MEDLINE, PsycINFO and CINAHL databases was undertaken with appropriate search terms, supplemented by reference searching. Published quantitative and qualitative primary research was included. Findings were reported in line with Preferred Reporting Items for Systematic Reviews and MetaAnalyses.

Result. Records identified through database searching 2,347; additional records 139; records following removal of duplicates 1,324. Full text studies included 41: 'Curriculum and Grading' $(\mathrm{n}=4)$; 'Mindfulness and Yoga' $(\mathrm{n}=11)$; 'Stress Management/Relaxation' $(\mathrm{n}=13)$; 'Behavioural Interventions' $(\mathrm{n}=3)$; 'Cognitive \& Selfawareness Interventions' $(\mathrm{n}=2)$; Mentorship $(\mathrm{n}=3)$; 'Education, Screening and Access to care' $(\mathrm{n}=3)$; 'Multifaceted Interventions' $(\mathrm{n}=2)$.

Effective interventions include those that reduce academic stress through grading changes and supporting transition to clinical training; resilience enhancing interventions such as mindfulness, yoga, CBT, group based exercise and relaxation; peer mentorship; faculty mentorship when actively engaged by the mentor; reducing stigma; improving detection; and improving access to treatment.

Outcomes for clinical year students were less promising, suggesting interventions may be insufficient to combat clinical stressors.

Conclusion. We propose a framework for implementing these effective interventions through 'Ecological and Preventative' paradigms. The former highlights an individual's interaction with their sociocultural environment, recognising multiple levels of influence on health: individual, interpersonal, institutional, community, and national. At each level the framework of primary, secondary and tertiary prevention can be applied.

Primary Prevention (intervening before health is impacted): reducing academic stress; resilience interventions; mentorship; peer support; brief interventions to avoid progress to established disorders.
Secondary Prevention (reducing prevalence of disorder): early detection through staff training and screening; treatment referral pathways; reciprocal arrangements if peers are placed within local settings.

Tertiary Prevention (reducing impairment): reasonable adjustments, communicated between placements

This recognises that medical students require a range of interventions at multiple levels to reduce stress, promote wellbeing and manage the spectrum of mental health difficulties they may encounter. The ecological framework also acknowledges the reciprocity of individuals being influenced by and influencing their environment, which aligns with the concept of co-production.

A systematic review of comparative time to all cause discontinuation of antipsychotic medications in first episode psychosis

\author{
Alice Brooke*, Richard Whale and Mihaela Bucur \\ Brighton and Sussex Medical School, Univeristy of Sussex, Sussex \\ Partnership NHS Foundation Trust (SPFT) \\ ${ }^{\star}$ Corresponding author.
}

doi: 10.1192/bjo.2021.642

Aims. Prompt treatment with medication and intensive psychosocial support are interventions that have been shown to improve function and prognosis in patients with First Episode Psychosis. NICE guidelines state that patients with a first episode of Psychosis should remain on an antipsychotic medication for 12 years to reduce risk of relapse, yet most patients stop long before 2 years. This systematic review explores the comparative time to all cause discontinuation of antipsychotics, often used as a marker of real-world treatment effectiveness, in First Episode Psychosis patients.

Method. A literature search was performed across multiple healthcare databases from 1980 to present day in the English Language. Inclusion criteria covered patients with a First Episode of Psychosis aged 14 years and over, and studies that were randomised controlled trials or observational in nature. The primary outcome measure was time to discontinuation of antipsychotic medication. Bias was assessed using the GRADE approach.

Result. 11 studies and 3840 patients were included in the review. Seven studies were randomised clinical trials; three were blinded, and four open-label. The remaining four were observational studies. All but one of the studies had a minimum follow-up period of one year (with a maximum of three years). Due to significant methodological heterogeneity across studies, it was not possible to perform meta-analysis. Narrative analysis of the results showed that Olanzapine performed ranked best, and wasbeing taken for the longest time period by patients, followed by Risperidone.

Conclusion. Multiple reviews exist on the efficacy of antipsychotics in First Episode Psychosis, but this is the first one to focus on time to discontinuation as a distinct outcome measure. The review encompasses a large sample size across North America, Eastern Asia and Europe. The interaction of time to discontinuation of antipsychotics with associated symptom levels and medication doses remains an area for further research. The review highlighted the significant differences in statistical methodology across studies in this emerging field, and the need for standardisation in ongoing research. Whilst effectiveness may therefore be greatest for olanzapine, this is outweighed in current guidance by its least favourable metabolic adverse effects profile. 